\title{
Localization of Blockchain and E-Currency Model for E-Government Services
}

\author{
Maryam Niknezhad * \\ Department of Information Technology Management, Central Tehran Branch, Islamic Azad University, Tehran, Iran \\ Maryam.Niknezhad@gmail.com \\ Sajjad Shokouhyar \\ Department of management, Shahid Beheshti University, Tehran, Iran \\ s_shokouhyar@sbu.ac.ir \\ Mehrzad Minouei \\ Department of management, Central Tehran Branch, Islamic Azad University, Tehran, Iran \\ meh.minouei@iauctb.ac.ir
}

Received: 04/Mar/2020 Revised: 24/Jul/2020 Accepted: 08/Sep/2020

\begin{abstract}
Blockchain can reduce bureaucracy and increase the efficiency and performance of administrative processes through a platform possessing features and attributes such as storing and exchanging electronic messages in a decentralized environment and executing high level of security transactions and transparency, if used in government public service delivery. Many scholars believe that this distributed technology can bring new utilizations to a variety of industries and fields, including finance and banking, economics, supply chain, and authentication and increase economic productivity and efficiency dramatically by transforming many industries in the context of today's economy. The present study, presents the characteristics of the localized blockchain and e-currency conceptual model for the evolution of e-government services. It also examines the impact of the blockchain and e-currency model on the economy and electronic financial transactions as a viable, practical and constructive solution (rather than blocking and filtering of e-currency and blockchain). Ultimately designing a localized block chain and e-currency model, has played an effective role in exploit its high potential to speed up the administrative processes and reduce costs related to electronic transactions and payments in e-government and increase e-government revenues and ultimately it can speed up the customer service delivery and increase their satisfaction with the government.
\end{abstract}

Keywords: Blockchain; E-Currency; E-Government; Distributed Ledger Technology; Artificial Intelligence.

\section{1- Introduction}

Today, society and the global economy are governed by the trust we have on intermediaries such as banks, governments, and big Internet companies like Google and Facebook. Some of the largest corporations and the greatest wealth come from becoming an intermediary in the business world. Intermediaries accomplish a transaction and take their share. Such intermediaries do a great job but have their own limitations: "they are costly and slow down everything. Anything that becomes central is vulnerable." But above all, they "make a disproportionate profit" of what they have provided. Simply put: for the little value they add, they make a lot of money. Their best product is trust, and that trust is built on the notion of their perpetual existence [1].

Decentralized systems face major problems, including scalability and issues related to privacy and multi-identity. So, experts are trying to design decentralized protocols that are attack resistance in addition to being scalable and optimized. Analyzing such protocols requires extensive knowledge in areas such as distributed systems, cryptography, game theory, and concepts of information theory. Blockchain technology basically could be regarded as a public ledger and all of committed transactions or digital events are stored in a list of blocks. This chain grows as new blocks are appended to it continuously. Asymmetric cryptography and distributed consensus algorithms have been implemented for user security and ledger consistency. The blockchain technology generally has key characteristics of decentralization, persistency, anonymity and auditability. With these traits, blockchain can greatly save the cost and improve the efficiency [2], [3] and facilitate the move to a more equitable and flat society. Today, more than 100 blockchain projects created to transform government systems are being conducted in more than 30 countries. What leads countries rapidly initiate blockchain projects? It is because blockchain is a technology directly related to social organization; unlike other technologies, a consensus mechanism form the core

\footnotetext{
* Corresponding Author
} 
of blockchain. Traditionally, consensus is not the domain of machines but rather humankind. However, blockchain operates through a consensus algorithm with human intervention; once that consensus is made, it cannot be modified or forged [4].

There are two different perspectives for governments in relation to the rise of blockchain architectures and applications. On the one hand the perspective of governance by blockchain, in which public organizations adopt blockchain technology for their own processes, like service provisioning, and in which blockchain technology is used to govern transactions. The other perspective is termed governance of blockchain, or blockchain Governance, which determines how blockchain should look like, how to adapt to changes and should ensure that public values and societal needs are fulfilled. Both require in-depth knowledge of the blockchain technology and the situation at hand [5].

In traditional e-government model Just as a common transaction, for example, need to experience more government audit and the corresponding archive, subsequent department needs through internal data query platform of leading department database data, and combining with affairs to deal with the data submitted to the department, offer certain audit opinion. Although the whole process has realized information, the system has the shortcomings like long business time between departments, low efficiency, lack of multiple levels of permissions on the laws and regulations, among database data redundancy is serious, the lack of unified update management, failure to ensure data security and high cost. But the egovernment system based on blockchain government affair has the advantages like high efficiency, build a multi-level laws and regulations of the authority, unified database and data security guaranteed. The application of blockchain technology to e-government also can reconfigure public resources, improve government efficiency, save cost, improve the basic income of people, and promote the construction of harmonious social relations [6].

With the increasing number of e-users in Iran and the increasing use of e-government services throughout the country, banking networks traffic as well as e-service centers are gradually increasing due to the use of traditional protocols and methods for money transfer and service provision. The use of blockchain and e-currency services can play a very effective role in accelerating and reducing the cost of financial transactions and electronic payment transfers in e-government, increasing egovernment revenues, reducing banking traffic, and speeding up delivery of service to customers. Providing a localized blockchain and e-currency model can also ensure security of service delivery and greatly prevent subversive attacks and theft of customer information and accounts. Obviously, to achieve this, the culture of using e-currency and e-services must be created and developed for the society and people, also trust and necessary substrates must be made.

Finally, the problem statement of this research can be the fatigue of decision-makers of E-Currency and EGovernment services due to the combination of different methods of reducing e-government relocation expenditure, Enhancing the security of e-government services, Blockchain capabilities to reduce the breakdown of the consensus protocol, increasing e-revenues of the government, and the use of e-currency based on consensus algorithms; in improving payment of e-government services. On the other hand, the need for using an intelligent system is in order to increasing confidence and reliability in decision making, as well as the need for multiple expertise by simultaneously utilizing the expertise of different field specialists to solving problems of research.

\section{2- Related Work}

According a study that analyses seven pilot blockchain deployments in the public sector in Europe, Significant incremental benefits can be realized in some areas through the utilization of blockchain technologies for the provision of public services. The two main groups of are increased security (enhancement of data integrity, immutability and data consistency between organizations) and efficiency gains (such as reduced processing time and lower costs). At this stage of the technology life cycle, the continuation of experimentation with different technical designs is vital. Prior to the scale-up, technical and governance standards need to be developed, in order ensure interoperability of different designs and facilitate operative services. Incompatibility between blockchain-based solutions and existing legal and organizational frameworks is a major barrier to unlock the transformative potential of blockchain Hence, the major policy objective should be to increase the technological and ecosystem maturity of distributed ledgers. Policy actions should aim not only at adaption of the technology to existing ecosystems but also at transformation of existing processes, organizations and structures using the disruptive potential of blockchain [7]. There are several working groups and pilot projects (in all stages of work ranging from proposed, to under development, to deploy) focused on applying blockchain within the U.S. government. The most common trends evaluated by federal agencies include: financial management, procurement, supply chain management, smart contracts, government-issued credentials, Federal personnel workforce data, Federal assistance programs, foreign aid delivery, health records and biometric data. But Increase technical understanding of blockchain within government by developing familiarity with the 
decentralized and distributed paradigms of DLT $^{1}$, Develop an internal blockchain subject matter expert workforce, Participate in the stewardship of blockchain and DLT by entering collaborative relationships with institutions like the World Economic Forum's Center for the Fourth Industrial Revolution, Increase government awareness of malign crypto-financial activity, Consider and study privacy \& legality implications especially regarding the intentional "right to be forgotten" and accidental private key destruction and Amplify knowledge of potential blockchain-based national security threats, particularly in intelligence, critical infrastructure, and the Internet of Things must be considered [8].

Blockchain technology as a type of decentralized transaction and data management technologies, provide trust, obscurity, security and data integrity without having to use any third party controlling organization. The literature review identify three groups of factors, namely institutional (norms and culture, regulations and legislations, governance), market (market structure, contracts and agreements, business process) and technical (information exchange and transactions, distributed ledges, shared infrastructure) those are needed for organizational adoption of blockchain. Factors presented in this framework (institutional factors, market factors and technical factors) interact and mutually influence each other. The way how different factors will interact with each other depends on the context in which blockchain will be adopted. Additionally, factors which influence the adoption of blockchain technologies depend on its intended use [9].

\section{2-1- Theoretical Framework and Variables}

Based on the evaluations and critical reviews of the books and articles related to the research model, at first, variables, indices, and measures are identified. The initial research model, the relationship between variables, and localization of the blockchain and e-currency model via this relationship for e-government payment are following specified:

\section{2-1-1- E-government Component [4], [10], [11], [12]:}

2-1-1-1-Reducing e-government relocation expenditure: Reducing e-government expenditure on hardware Reducing e-government expenditure on software Reducing e-government expenditure on human resources

2-1-1-2- Enhancing the security of e-payment services of e-government:

Protecting user information of personal accounts Improving the security level of users' accounts

\footnotetext{
${ }^{1}$ Distributed Ledger Technology
}

Preventing hacking and intrusion into the e-service system network of the e-government

2-1-1-3- Increasing e-revenues of the government:

Increase of government e-revenues in G2B transactions

Increase of government e-revenues in G2G transactions

Increase of government e-revenues in G2C transactions

2-1-2- E-Currency Application Component [10], [13], [3], [14]:

2-1-2-1- The international value of e-currency: International consensus on the value of currency The value of currency based on the reduction of Internet banking network traffic

The value of currency based on the type

2-1-2-2- The volume of e-currency:

The volume of currency available to individuals

The volume of currency available to businesses

The volume of currency available to governments

2-1-2-3- E-currency rules:

Managing the implementation of contracts related to the selection and qualification of supervisory and operating parties

Developing and communicating the technical architecture of e-services through blockchain and e-currency

Management of providing consulting, educational, and cultural services to the executives

2-1-3- Blockchain Capabilities Component [4], [3], [15], [16]:

2-1-3-1- Decentralization capability:

Distributed data logging

Distributed data storage

Updating data as distributed

2-1-3-2- Open-source capability:

Developing of applications by people

Evaluating the data publicly

Transparency of data and applications for people

2-1-3-3- Anonymity capability:

Anonymity of data transfer

Anonymity of transaction

Increase of trust between nodes

2-1-3-4- Independence capability:

Independent data transfer

Independent data updating

Protection and immutability of all records forever

Figure 1 shows the initial model of research and the relationship between variables.

After reviewing the theoretical foundations of the research and investigating the literature history of the research, it was determined that considering the research gaps in the knowledge domains of "reducing e-government relocation expenditure, Enhancing the security of e-government 
services, Blockchain capabilities to reduce the breakdown of the consensus protocol, increasing e-revenues of the government, and the use of e-currency based on consensus algorithms; in improving payment of e-government services", as well as the lack of an intelligent system to provide guidance to the managers for decision making, the research innovations are localizing and realized in solving those research gaps.

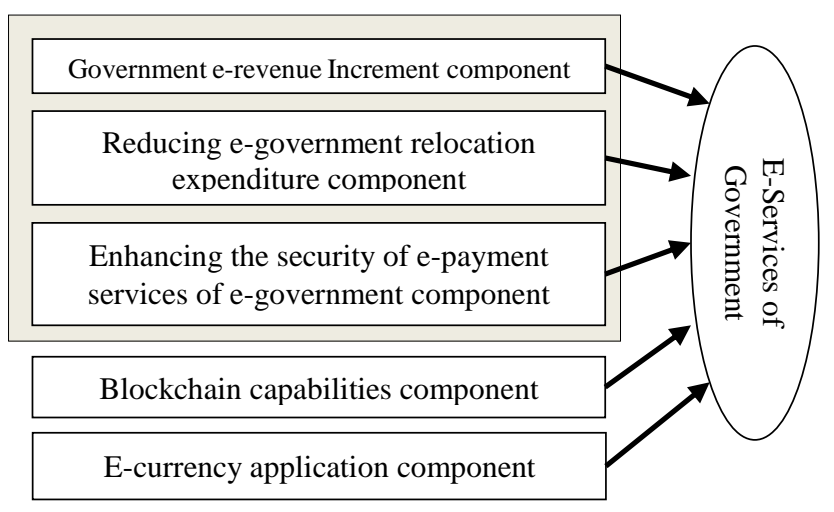

Fig. 1 Initial model of research and the variables.

\section{2-2- Data Analysis Method}

After reviewing the research background and theoretical foundations of the research, it was found that no similar research has been carried out to localize the blockchain and e-currency model for e-government services based on the documentation of e-government services of the country, employing a combined methodology of statistical analysis and artificial intelligence in MATLAB.

Figure 2 illustrates the research steps as following. A descriptive-modeling and exploratory (qualitative, quantitative) type of research was conducted. Due to the use of articles and documentation related to the research subject from a variety of sources, the method of data collection in this research is a "case-study of documentation". To evaluate the rules of artificial intelligence system based on the artificial neural network to localize the model extracted from expert opinions, the tools of determining the variables of decision-making model and interview have been utilized.

The study population consisted of professors, specialists, and experts working in the Iranian Blockchain Association and the Blockchain Laboratory of the Sharif University of Technology in Iran or similar positions. The sampling method is a combination of two methods of nonprobability purposive sampling (judgmental) and snowball sampling. Due to the nature of the sampling method, the sample size of the study will be equal to the number of available and collaborative experts.

Consensus algorithms (especially Proof-of-Work (POW) and Proof-of-Stake (POS)) are used to present and evaluate the localized model. Also, breakdown analysis was used to investigate the intrusion and weaknesses of the localized model. In addition, to investigate the security of the proposed model against hackers and subversive attacks, the randomly chosen nodes analysis method in the network is used to create blocks by exploiting both Nakamoto and voting methods.

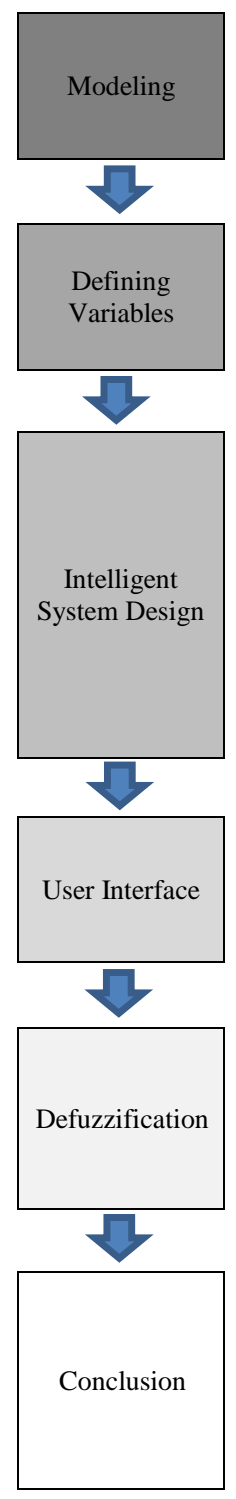

Modeling of e-government concepts to identify input and output variables and to draw relationships between them (with input-output approach)

Defining qualitative variables using linguistic constraints and assigning them numbers, smart sets, and membership functions (using triangular and trapezoidal smart numbers)

Introducing intelligent inference system using toolkit of artificial neural networks of MATLAB programming environment: this step involves extracting expert rules and evaluating them by experts and creating an intelligent base of rules as well as designing inference engine with access to intelligent rules (using toolkit of artificial neural networks of MATLAB programming environment )

User interface design, displaying options, and the way to use intelligent inference system using toolkit of artificial neural network programming environment designed in MATLAB

Selecting a method for defuzzification to convert numbers and smart sets to a definite value to check for actual system performance (using toolkit of artificial neural network programming environment of MATLAB)

Analysis of intelligent inference system outputs for localization analysis of blockchain model and e-money for e-government payment using toolkit of artificial neural network programming environment of MATLAB (with system analysis approach)

Fig. 2 Flowchart of research steps

\section{3- Research Methodology: Intelligent System Design Based on Artificial Neural Networks}

The methodology of this article is applied-modeling because it aims to accurately describe the concepts and rules related to the Blockchain and E-Currency model for E-Government services. On the other hand, the 
relationship between these concepts and rules is assessed and evaluated by the experts. Indeed, Statistical Analysis is the study of the effect of input variables on the output variable at a statistical model.

The Research technique of this article is Artificial Neural Networks in Matlab software. One of the most important reasons for using artificial neural networks and fuzzy systems in this research is that real world issues typically have a complex structure, which implies ambiguity and uncertainty in their definition and understanding [17], [18]. Ever since it has been able to think, it has always been ambiguous in various social, technical and economic issues. The human brain defines and evaluates sentences by considering various factors based on inferential thinking, whose pattern in mathematical language and formulas, if not impossible, will be very complicated [17], [19]. Linguistics variables are expressed on the basis of language (spoken) values that are in the phrase set (words / terms), and language expressions are attributes for linguistic variables. Here, linguistic variables are said to be variables that words, and sentences of human and machine languages are acceptable values for them instead of numbers. A fuzzy number is a special fuzzy set in which $\mathrm{x}$ denotes the true values of the member of the set of $\mathrm{R}$ and its membership function $\mu_{\widetilde{A}}(x)$ as it is Eq. (1) [17], [18]:

$$
\mathrm{A}^{\prime}=\left\{\left(\mathrm{x}, \mu_{\tilde{A}}(x)\right) \mid \mathrm{x} \in \mathrm{X}\right\}
$$

In fact, the dividing below describes how the relationship between fuzzy logic and the artificial neural network is expressed in terms of this view [17], [18], [19]:

Symmetric Neuro-Fuzzy models: The neural network and the fuzzy system work on one single operation, but they do not affect each other. None of them are used to determine another parameter. Usually, in this model, the neural network is used to pre-process the input or output of the fuzzy system.

Artificial Neural Network based Fuzzy Inference Systems: Some of these systems are considered as Cooperative models. These models are used to expand fuzzy rules.

Combined Neuro-Fuzzy models: Artificial neural network and fuzzy system combine in a coordinated structure. This pattern can be considered as a neural network with a fuzzy parameter or a distributed learning fuzzy system. ANFIS and ANNBFIS are examples of this model.

Finally, the five steps of designing an intelligent system based on artificial neural networks to localize the blockchain and e-currency model for payment of egovernment services according to Figure 3 are as follows:

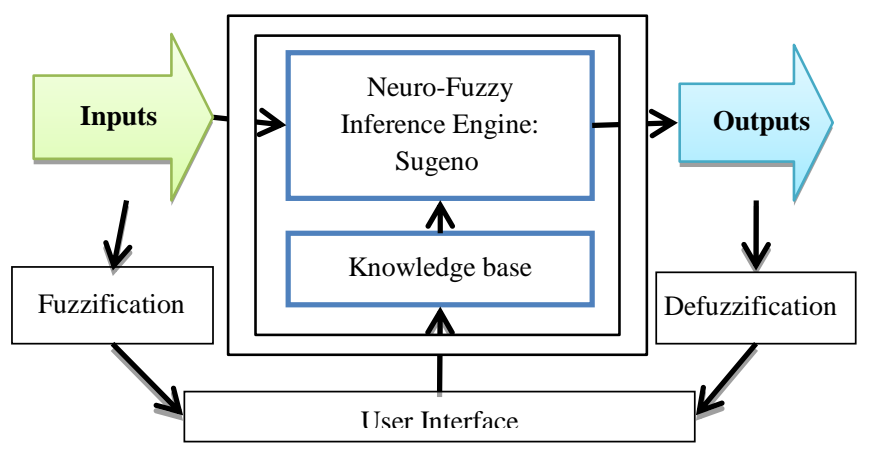

Fig. 3 Intelligent System Structure Based on artificial neural networks

Step 1: Modeling of the field concepts to identify input and output variables and to draw relationships between them

Step 2: Defining qualitative variables, exploiting linguistic constraints and assigning them numbers, fuzzy sets, and membership functions

Step 3: Designing an intelligent system based on artificial neural networks including the extraction of expert rules, their evaluation by experts, the creation of fuzzy rules database, and designing inference engine with access to fuzzy rules.

Step 4: Designing user interface, displaying options, and using the designed intelligent system

Step 5: Selecting a method for defuzzification to convert fuzzy numbers to a definite value to verify the actual performance of the system.

The sample and population of this research can be divided into two general groups: the first group consists of University Professors (Academic Experts), the second group includes experts working in e-government services or similar positions (Industrial Experts).

\section{4- Results}

After distributing 100 questionnaires, the sample size of this study is 96 available and cooperative experts who were selected by a combination of two methods: nonprobability purposive (judgmental) sampling and snowball sampling. The data related to measure 1 (localization tool of blockchain model and e-currency for paying egovernment services) and measure 2 (validation tool of the intelligent system) were collected in the fall of 2019. Table 1 shows the descriptive information of the research variables and indicators, based on the number of data and mean, and standard deviation, indicating that the data in this study are in good condition in terms of symmetry and aggregation. The most important criteria for the variables are outlined in the table. 
Table 1: descriptive information of the research variables and indicators

\begin{tabular}{|c|c|c|c|c|}
\hline \multirow{2}{*}{ Variables and Indicators } & \multicolumn{2}{|c|}{ Ideal } & \multicolumn{2}{|c|}{$\begin{array}{l}\text { Functional } \\
\text { status }\end{array}$} \\
\hline & Data & Mean & Data & Mean \\
\hline $\begin{array}{l}\text { Reducing e-government } \\
\text { relocation expenditure (X1) }\end{array}$ & 96 & 5.47 & 92 & 4.90 \\
\hline $\begin{array}{c}\text { Reducing e-government } \\
\text { expenditure on human resources }\end{array}$ & 92 & 5.65 & 96 & 4.21 \\
\hline $\begin{array}{l}\text { Reducing e-government } \\
\text { expenditure on infrastructure }\end{array}$ & 96 & 5.63 & 96 & 4.66 \\
\hline $\begin{array}{l}\text { Reducing e-government } \\
\text { expenditure on media }\end{array}$ & 96 & 5.68 & 92 & 4.59 \\
\hline $\begin{array}{l}\text { Reducing e-government } \\
\text { expenditure on hardware }\end{array}$ & 96 & 5.80 & 96 & 4.76 \\
\hline $\begin{array}{l}\text { Reducing e-government } \\
\text { expenditure on software }\end{array}$ & 96 & 5.91 & 96 & 4.25 \\
\hline $\begin{array}{l}\text { Enhancing the security of e- } \\
\text { government services (X2) }\end{array}$ & 96 & 5.79 & 96 & 5.05 \\
\hline $\begin{array}{l}\text { Preventing hacking and intrusion } \\
\text { into the e-service system } \\
\text { network of the e-government }\end{array}$ & 96 & 5.51 & 94 & 4.61 \\
\hline $\begin{array}{l}\text { Information Security } \\
\text { Management System }\end{array}$ & 96 & 5.67 & 92 & 4.79 \\
\hline $\begin{array}{l}\text { Protecting user information of } \\
\text { personal accounts }\end{array}$ & 92 & 5.93 & 96 & 4.48 \\
\hline $\begin{array}{c}\text { Improving the security level of } \\
\text { users' accounts }\end{array}$ & 96 & 5.92 & 94 & 4.67 \\
\hline $\begin{array}{c}\text { Encryption of e-payment } \\
\text { services }\end{array}$ & 96 & 5.77 & 96 & 4.91 \\
\hline $\begin{array}{l}\text { Blockchain capabilities to reduce } \\
\text { the breakdown of the consensus } \\
\text { protocol (X3) }\end{array}$ & 96 & 5.73 & 90 & 5.31 \\
\hline Anonymity capability & 96 & 5.91 & 96 & 5.01 \\
\hline Decentralization capability & 92 & 5.95 & 94 & 4.76 \\
\hline Independence capability & 96 & 5.51 & 92 & 4.90 \\
\hline Open-source capability & 96 & 5.62 & 92 & 4.52 \\
\hline Immutability capability & 96 & 5.66 & 96 & 4.95 \\
\hline $\begin{array}{l}\text { Increasing e-revenues of the } \\
\text { government (X4) }\end{array}$ & 96 & 5.56 & 92 & 4.95 \\
\hline $\begin{array}{l}\text { Increase of government e- } \\
\text { avenues in } \mathrm{G} 2 \mathrm{G} \text { transactions }\end{array}$ & 96 & 5.67 & 92 & 4.39 \\
\hline $\begin{array}{l}\text { Increase of government e- } \\
\text { avenues in G2B transactions }\end{array}$ & 96 & 5.83 & 96 & 4.42 \\
\hline $\begin{array}{l}\text { Increase of government e- } \\
\text { avenues in } \mathrm{B} 2 \mathrm{~B} \text { transactions }\end{array}$ & 96 & 5.68 & 96 & 4.58 \\
\hline $\begin{array}{l}\text { Increase of government e- } \\
\text { avenues in G2C transactions }\end{array}$ & 96 & 5.76 & 92 & 4.30 \\
\hline
\end{tabular}

\begin{tabular}{|c|c|c|c|c|}
\hline \multirow{2}{*}{ Variables and Indicators } & \multicolumn{2}{|c|}{ Ideal } & \multicolumn{2}{c|}{$\begin{array}{c}\text { Functional } \\
\text { status }\end{array}$} \\
\cline { 2 - 5 } & Data & Mean & Data & Mean \\
\hline $\begin{array}{c}\text { Increase of government e- } \\
\text { avenues in B2C transactions }\end{array}$ & 96 & 5.59 & 96 & 4.82 \\
\hline $\begin{array}{c}\text { use of e-currency based on } \\
\text { consensus algorithms (X5) }\end{array}$ & 96 & 5.73 & 96 & 4.63 \\
\hline $\begin{array}{c}\text { e-currency rules based on PoW } \\
\text { Type of e-currency based on PoS }\end{array}$ & 96 & 5.52 & 96 & 4.76 \\
\hline $\begin{array}{c}\text { Liquidity based on PoW } \\
\text { International Value of e-currency } \\
\text { Based on PoW }\end{array}$ & 96 & 5.61 & 94 & 4.61 \\
\hline $\begin{array}{c}\text { Volume of e-currency based on } \\
\text { PoS }\end{array}$ & 96 & 5.91 & 94 & 4.86 \\
\hline
\end{tabular}

Following are the five steps of designing and implementing of the intelligent system for localizing the model:

Step one: input and output variables are defined. Input variables of the intelligent system involve reducing egovernment relocation expenditure (X1), Enhancing the security of e-government services (X2), Blockchain capabilities to reduce the breakdown of the consensus protocol (X3), increasing e-revenues of the government (X4), and the use of e-currency based on consensus algorithms (X5); the output variable of the intelligent system is the status of "improving payment of egovernment services."

Step two: qualitative variables are defined by linguistic constraints and assigning them numbers, fuzzy sets, and membership function. Table 2 illustrate the linguistic variables, fuzzy values, and membership functions of triangular and trapezoidal numbers associated with the input and output variables of the intelligent system within three- and five-spectra.

Table 2: linguistic variables associated with the input and output variables of the intelligent system

\begin{tabular}{|c|c|}
\hline \multirow{2}{*}{ Linguistic variables } & $\begin{array}{c}\text { Membership functions of } \\
\text { triangular and trapezoidal } \\
\text { numbers }\end{array}$ \\
\hline Low & $(0.30 .150)$ \\
\hline Medium & $(0.70 .50 .3)$ \\
\hline High & $(10.850 .7)$ \\
\hline \multicolumn{2}{|c|}{ Training Data (ANFIS) } \\
\hline \multicolumn{2}{|c|}{$0,0,0,0,0,0$} \\
\hline \multicolumn{2}{|c|}{$0-0.025,0-0.025,0-0.025,0-0.025,0-0.025,0.05$} \\
\hline
\end{tabular}




\begin{tabular}{|c|}
\hline $0.025-0.05,0.025-0.05,0.025-0.05,0.025-0.05,0.025-0.05,0.1$ \\
\hline $0.05-0.075,0.05-0.075,0.05-0.075,0.05-0.075,0.05-0.075,0.15$ \\
\hline $0.075-0.10,0.075-0.10,0.075-0.10,0.075-0.10,0.075-0.10,0.2$ \\
\hline $0.10-0.15,0.10-0.15,0.10-0.15,0.10-0.15,0.10-0.15,0.25$ \\
\hline $0.15-0.25,0.15-0.25,0.15-0.25,0.15-0.25,0.15-0.25,0.3$ \\
\hline $0.25-0.30,0.25-0.30,0.25-0.30,0.25-0.30,0.25-0.30,0.35$ \\
\hline $0.30-0.35,0.30-0.35,0.30-0.35,0.30-0.35,0.30-0.35,0.4$ \\
\hline $0.35-0.4,0.35-0.4,0.35-0.4,0.35-0.4,0.35-0.4,0.45$ \\
\hline $0.40-0.45,0.40-0.45,0.40-0.45,0.40-0.45,0.40-0.45,0.5$ \\
\hline $0.45-0.5,0.45-0.5,0.45-0.5,0.45-0.5,0.45-0.5,0.55$ \\
\hline $0.50-0.55,0.50-0.55,0.50-0.55,0.50-0.55,0.50-0.55,0.6$ \\
\hline $0.55-0.6,0.55-0.6,0.55-0.6,0.55-0.6,0.55-0.6,0.65$ \\
\hline $0.60-0.65,0.60-0.65,0.60-0.65,0.60-0.65,0.60-0.65,0.7$ \\
\hline $0.65-0.7,0.65-0.7,0.65-0.7,0.65-0.7,0.65-0.7,0.75$ \\
\hline $0.70-0.75,0.70-0.75,0.70-0.75,0.70-0.75,0.70-0.75,0.8$ \\
\hline $0.75-0.80,0.75-0.80,0.75-0.80,0.75-0.80,0.75-0.80,0.85$ \\
\hline $0.8-0.85,0.8-0.85,0.8-0.85,0.8-0.85,0.8-0.85,0.9$ \\
\hline $0.85-0.9,0.85-0.9,0.85-0.9,0.85-0.9,0.85-0.9,0.925$ \\
\hline $0.90-0.95,0.90-0.95,0.90-0.95,0.90-0.95,0.90-0.95,0.95$ \\
\hline $0.95-1,0.95-1,0.95-1,0.95-1,0.95-1,0.975$ \\
\hline $1,1,1,1,1,1$ \\
\hline
\end{tabular}

Step three: a knowledge base of intelligent system is designed, which involves extracting expert rules, evaluating them by experts, and creating fuzzy rules database. The starting point of building a rule-based knowledge base is to obtain a set of rules when a phase of expert knowledge or the field of knowledge being examined and the subsequent step are a combination of these rules into a single system. Finally, the number of fuzzy rules of the module "improving the payment of egovernment services in the country" of the intelligent system is equal to 243 because of the five main variables, each of which has three states. Figure 4 shows how to generate fuzzy rules within the knowledge base.

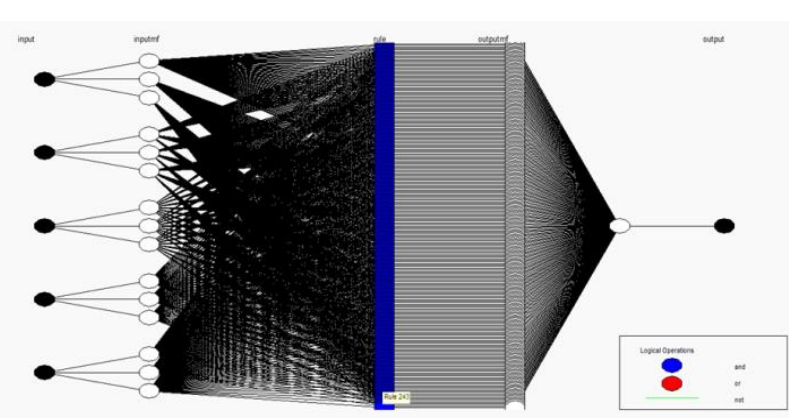

Fig. 4 How to generate fuzzy rules inside the knowledge base
Step four: an inference engine of intelligent system is designed (Figure 5). In this step, the wtaver method is used for defuzzification to convert fuzzy numbers and sets to a definite value for actual evaluation of the system performance

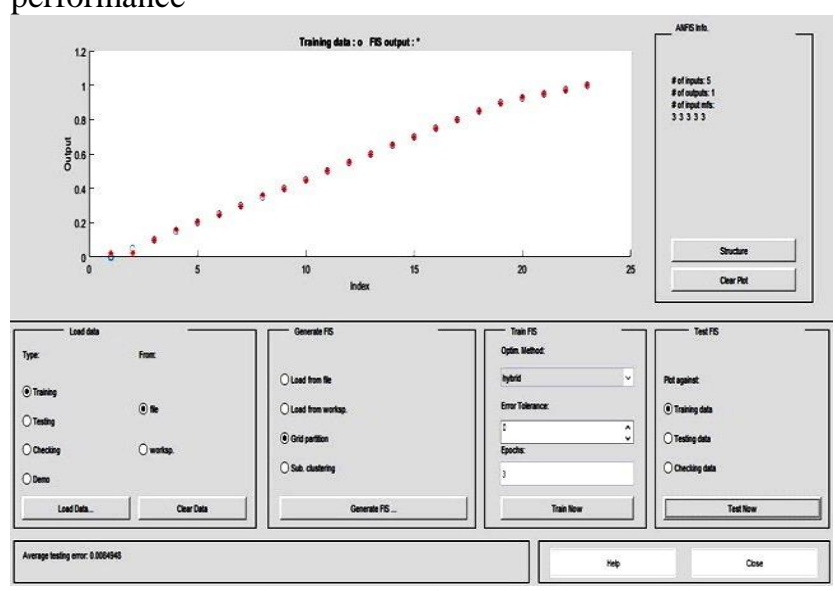

Fig. 5 System Inference Engine

The mean error of the test data was calculated 0.0085 (less than $1 \%$ ) in the inference engine of the intelligent system for "localization of blockchain and e-currency model for the payment of e-government services", which shows the high accuracy of the calculations of artificial neural networks of the research. The defuzzification in the intelligent system converts the fuzzy output to a definite number.

Step five: this step explains how to exploit the intelligent system and analyze its outputs numerically (accurate) and linguistically to analyze the behavior of system's output variable. In order to determine the weight of the input values of the system, information about the ideal and functional weight of each of the main variables of the research is presented in Table 3.

Table 3: Ideal weight and functional weight of main variables

\begin{tabular}{|c|c|c|c|c|}
\hline \multirow{2}{*}{ Variables } & \multicolumn{2}{|c|}{ Ideal weight } & \multicolumn{2}{c|}{ Functional weight } \\
\cline { 2 - 5 } & $\begin{array}{c}\text { Weighted } \\
\text { average }\end{array}$ & $\begin{array}{c}\text { Fuzzy } \\
\text { Weight }\end{array}$ & $\begin{array}{c}\text { Weighted } \\
\text { average }\end{array}$ & $\begin{array}{c}\text { Fuzzy } \\
\text { Weight }\end{array}$ \\
\hline (X1) & 5.690 & 0.813 & 4.562 & 0.652 \\
\hline$(\mathrm{X} 2)$ & 5.765 & 0.824 & 4.752 & 0.679 \\
\hline$(\mathrm{X} 3)$ & 5.730 & 0.819 & 4.903 & 0.701 \\
\hline (X4) & 5.682 & 0.812 & 4.577 & 0.654 \\
\hline (X5) & 5.703 & 0.815 & 4.683 & 0.669 \\
\hline
\end{tabular}

According to the rules of knowledge base of the main module of the intelligent system and based on the calculation of the weight of each main variable using the expert opinions and also taking advantage of the intelligent system based on designed artificial neural networks, it is 
possible to numerically and more precisely examine the status of "improving the payment of e-government services in the country"

The main findings of research based on localized model is utilizing intelligent system outputs, the status of "improving the payment of e-government services of the country" can be analyzed on the basis of variables such as "Blockchain capabilities to reduce breakdown of consensus protocol (X3)", "Increasing e-revenues of the government (X4)", "Enhancing the security of egovernment services (X2)", "Use of e-currency based on consensus algorithms (X5)", and "Reducing e-government relocation expenditure (X1)" because, as outlined in the EGovernment Foresight Framework, the services should at least be provided through only one single port. Or, in the most optimistic way, log-in should be via a port, and then the user transfer operation should be done.

In fact, according to the rules of knowledge base of the main module of the intelligent system based on the calculation of the weight of each main variable using the expert opinions and utilizing the intelligent system designed in this research, the status of "improving the payment of e-government services of the country" can be investigated numerically and more precisely: Ideally, if the status of "Reducing e-government relocation expenditure (X1)" is good, i.e. exactly 0.813, and "Enhancing the security of e-government services (X2)" is good, i.e. exactly 0.824, and "blockchain capabilities to reduce breakdown of consensus protocol (X3)" is good, i.e. exactly 0.819 , and "Increasing e-revenues of the government (X4)" is good, i.e. exactly 0.812, and "Use of e-currency based on consensus algorithms (X5)" is good, i.e. exactly 0.815 , then, "improvement of e-government services payment" is excellent (fifth level)", i.e. exactly 0.952. Considering the membership functions of linguistic variables provided by the experts in the previous tables, the value of 4.76 within a 5-value spectrum is calculated in a defined range for the linguistic variable "excellent", i.e. the improvement of the country's e-government payment services, with programming code of $[0.815 ; 0.812 ; 0.819$; $0.824 ; 0.813$ ] exactly $0.952 \quad(95 \%$ objective function optimality). On the other hand, in terms of performance status, if the status of "Reducing e-government relocation expenditure (X1)" is average, i.e. exactly 0.652, and "Enhancing the security of e-government services (X2)" is average, i.e. exactly 0.679 , and "Blockchain capacity to reduce breakdown of consensus protocol (X3)" is good, i.e. exactly 0.701 , and "Increasing e-revenues of the government (X4)" is average, i.e. exactly 0.654, and "Use of e-currency based on consensus algorithms (X5)" is average, i.e. exactly 0.669 , then, the status of "improvement of e-government services payment" is average (third level)", i.e. exactly 0.468 . According to the membership functions of linguistic variables provided by the experts in the previous tables, the value of 2.34 within a 5-value spectrum is calculated exactly 0.468 in a defined range for the linguistic variable "average", i.e. the status of the improvement of the country's e-government payment services, with programming code of $[0.669 ; 0.654 ; 0.701$; $0.679 ; 0.652]$.

After designing, the outputs and responses of the intelligent system were compared in a separate measurement tool with the opinions of 18 so-called experts, the results of which can be seen in Table 4 based on the intelligent system rules and the mean of expert responses. Since experts' opinions are expressed based on the spectrum of five membership functions, to test the hypothesis above, we can exploit the discrepancy percentage between the outputs of the intelligent system of this research and the mean of expert opinions.

Hence, the final difference between the outputs of the intelligent system and the mean of expert opinions was not significant and was equal to 0.065 . Since there is not a sufficient reason to accept the null hypothesis, the opposite hypothesis is accepted, i.e. there is no significant difference between the mean of expert opinions and the outputs of the "intelligent system".

Table 4: Comparison of "intelligent system" outputs with the mean of

\begin{tabular}{|c|c|c|c|}
\hline $\begin{array}{l}\text { Intelligent } \\
\text { system rules }\end{array}$ & $\begin{array}{c}\text { Intelligent } \\
\text { system } \\
\text { outputs }\end{array}$ & $\begin{array}{l}\text { Mean of expert } \\
\text { responses }\end{array}$ & Difference ratio \\
\hline Rule. 3 & 1 & 1.22 & $0.055=0.22 / 4$ \\
\hline Rule. 45 & 3 & 2.72 & $0.0675=0.28 / 4$ \\
\hline Rule. 79 & 3 & 2.78 & $0.055=0.22 / 4$ \\
\hline Rule. 86 & 2 & 1.67 & $0.0825=0.22 / 4$ \\
\hline Rule. 103 & 2 & 1.67 & $0.0825=0.22 / 4$ \\
\hline Rule. 140 & 3 & 2.78 & $0.055=0.22 / 4$ \\
\hline Rule. 157 & 3 & 3 & $0=0 / 4$ \\
\hline Rule. 219 & 2 & 2.94 & $0.015=0.06 / 4$ \\
\hline Rule. 224 & 2 & 2.39 & $0.1525=0.61 / 4$ \\
\hline Rule. 235 & 2 & 2.67 & $0.0825=0.33 / 4$ \\
\hline \multicolumn{3}{|c|}{ Final Difference } & 0.065 \\
\hline
\end{tabular}

\section{5- Conclusions}

One of the most important results of the research is that in improving the payment of e-government, blockchain technology has the ability to facilitate direct interaction between government agencies, citizens and economic actors, which at the basic level means improving public services in registration and information exchange processes.

Finally, utilizing the results of the present study, we may contribute to the removal of existing barriers to improvement of the payment of e-government services. 
The following solutions can facilitate the achievement of e-government's goals:

- Making Citizens' Digital Authentication more secure, for Enhancing the security of e-government services.

- Increasing citizens' ownership and control over economic processes, for increasing e-revenues of the government.

- Using smart contracts in process automation or registering economic documents in official government offices, for reducing e-government relocation expenditure.

- Implementing the Blockchain capabilities to reduce the breakdown of the consensus protocol, for the use of e-currency based on consensus algorithms.

- Supporting administrative agency to accelerate the electrification of their services.

- Using the potentials of the private enterprises to increase citizens' satisfaction with services.

- Reducing the duties of the government and transferring them to non-state sectors through the capabilities of information technology.

- Accelerate and facilitate the receipt of citizens and business's services from the executive apparatus.

- Creating a comprehensive and scientific model based on local solutions in e-services.

- Increasing the productivity of government agencies and reducing the cost of service exposition.

Also the most important recommendations and suggestions for future research can be stated as:

- Utilization of Data envelopment analysis (DEA) Methodology for measuring the efficiency of multiple decision-making units (DMUs) in the model.

- Using System Dynamic Methodology (Vensim) for relationship building modeling,

- Utilization of fuzzy Multiple Criteria Decision Making (MCDM) techniques for relationship network ranking in the model

- Employment of Fuzzy Ontology for the comprehensive modeling of relationships in the model.

\section{References}

[1] D. Tapscott and A. Tapscott, "Block chain revolution", Portfolio: Reprint edition, 2018, ISBN-10: 1101980141.

[2] Z. Zheng, S. Xie, H. Dai, X. Chen and H. Wang, “An overview of blockchain technology: Architecture, consensus, and future trends", in 2017 IEEE International Congress on Big Data (BigData Congress), 2017, DOI: 10.1109/ BigDataCongress .2017.85, pp. 557-564.

[3] W. Petersz, E. Panayiy, and A. Chapelley, "Trends in cryptocurrencies and blockchain technologies: A monetary theory and regulation perspective Gareth", arXiv: 1508.04364v1, [cs.CR], 2015.

[4] M. Jun, "Blockchain government - a next form of infrastructure for the twenty-first century", CreateSpace Independent Publishing Platform; 1 edition, 2018, ISBN-10: 171912714X, pp. 2-6.

[5] S. Olnes, J. U-b.acht and M. Janssen, "Blockchain in government: Benefits and implications of distributed ledger technology for information sharing", Government Information Quarterly, 34, Issue 3, 2017, pp. 355-364.

[6] H. Chen, "An E-government Model Design Based on Block Chain", in International Conference on Manufacturing Construction and Energy Engineering, 2017, ISBN: 978-160595-483-7, pp. 176-179.

[7] D. Allessie, M. Sobolewski and L. Vaccari, "Blockchain for digital government", Science for Policy report by the Joint Research Centre (JRC), the European Commission's science and knowledge service, 2019, ISBN 978-92-76-00582-7, doi:10.2760/93808. pp. 65-67.

[8] G. Mark, N. Melinda, P. Lisa, G. Bell, J. Downing, K. Rahbari and M. Kilani, "Blockchain and Suitability for Government Applications", PUBLIC-PRIVATE ANALYTIC EXCHANGE PROGRAM, 2018, pp. 27-31.

[9] M. Janssen, V. Weerakkody, E. Ismagilova, U. Sivarajah and Z. Irani, "A framework for analysing blockchain technology adoption: Integrating institutional, market and technical factorsInternational Journal of Information Management, 50, 2020, ISBN 0268-4012, pp.302-309.

[10] R. Böhme, N. Christin, B. Edelman, and T. Moore, "Bitcoin: Economics, Technology, and Governance", Journal of Economic Perspectives, 29, Number 2, 2015, pp. 213-238.

[11] E. Shahghasemi, B. Tafazzoli, M. Akhavan, G. Mirani and T. Khairkhah, "Electronic Government in Iran: A Case Study", Online Journal of Social Sciences Research. ISSN 2277-0844; Volume 2, Issue 9, 2013, pp. 254-262.

[12] A. Jahangiri, and N. Alavi, "Creating the Groundwork for an Electronic Government", Journal of Management and Development Process, 20 (3 and 4): 42-53, 2006. [In Persian]

[13] S. Barber, X. Boyen, E. Shi, and E.Uzun, "Bitter to BetterHow to Make Bitcoin a Better Currency", 16 th International Conference on Financial Cryptography and Data Security, 2012, pp. 399-414.

[14] E. Rasolinejad "Factors affecting the use of e-money: The Case of Saderat Bank", Journal of New Economy and Commerce, Volume 3, Issue 10, 2009, pp. 131-153. [In Persian]

[15] T. Aste, P. Tasca, and T.D. Matteo, "Blockchain Technologies: foreseeable impact on industry and society", COMPUTER, 50(9), 2017, pp. 18-28. 
[16] I. Lin and T. Liao, "A Survey of Blockchain Security Issues and Challenges", International Journal of Network Security, Vol.19, No 5, 2017, pp. 653-659.

[17] C. T. Lin and C. S. George Lee, Neural Fuzzy Systems: A Neuro-Fuzzy Synergism to Intelligent Systems, Hardcover Publisher: Prentice Hall; Har/Dskt edition, ISBN 10: 0132351692ISBN 13: 9780132351690, 1996.

[18] R. N. Mishra and K. Mohanty, "Real time implementation of an ANFIS-based induction motor drive via feedback linearization for performance enhancement", Engineering Science and Technology an International Journal, 2016, 19(4) DOI: 10.1016/j.jestch.2016.09.014

[19] S. Moayer and P. A. Bahri, "Hybrid intelligent scenario generator for business strategic planning by using ANFIS" Expert Systems with Applications, 2009, Volume 36, Issue 4, May 2009, pp. 7729-7737.

Maryam Niknezhad received the B.Sc. degree in computer engineering from Alzahra University of Tehran, Iran, in 2004. She received the M.Sc. degree in Information Technology engineering from Shiraz University, Iran, in 2012. Currently she is Ph.D. Candidate in Islamic Azad University, Central Tehran Branch, Iran. She works in Ministry of Economic Affairs and Finance, Iran. Her area research interests include Data mining, Social Commerce, Blockchain, Social Networking, and Deep Learning.

Sajjad Shokouhyar is an Assistant Professor of industrial management and information system at Shahid Beheshti University. He earned his doctoral degree in industrial engineering from Polytechnic University of Tehran and completed his M.S. in systems engineering at Polytechnic University. His research is focused on developing decision analytic tools that can be implemented in supply chain management, logistic, Transportation and supplier evaluation systems.

Mehrzad Minouei is an Assistant Professor of Financial Management at Central Tehran Branch, Islamic Azad University, Tehran, Iran. He received his Ph.D. degree in Financial Management from Science and Research Branch, Islamic Azad University, Iran. He is conducting research activities in the areas of Financial Management and Capital Market. 